\title{
Effects of drain quiescent voltage on the ageing of AlGaN/GaN HEMT devices in pulsed RF mode
}

\author{
A. Divay ${ }^{\mathrm{a}}$, C. Duperrier ${ }^{\mathrm{b}}$, F. Temcamani ${ }^{\mathrm{c}}$, O. Latry ${ }^{\mathrm{a}}$ \\ ${ }^{a}$ Normandie Univ, UNIROUEN, INSA Rouen, CNRS, Groupe de Physique des Matériaux, 76000 Rouen, France \\ ${ }^{b}$ University of Cergy, ETIS UMR 8051 CNRS, ENSEA, 95000 Cergy-Pontoise, France \\ ${ }^{c}$ Quartz, ENSEA, 95000 Cergy-Pontoise, France
}

\begin{abstract}
The reliability of RF AlGaN/GaN HEMT devices on $\mathrm{SiC}$ substrate is investigated here in pulsed RF condition at nominal and maximum rating drain quiescent bias. During these 3500 hours tests, high voltage especially during the RF pulse leads to a progressive decrease in $g_{m}$ and $I_{D S S}$ while trap concentration increases. These evolutions may be attributed to trap generation by hot carrier injection and highlight the importance of drain quiescent voltage as an important acceleration factor for this technology's reliability in pulsed RF conditions.
\end{abstract}

Keywords: GaN, HEMT, ageing tests, reliability, traps, transconductance

\section{Introduction}

The gain in power density and thermal dissipation make the AlGaN/GaN HEMT (High Electron Mobility Transistors) technology a promising candidate for power applications (radiofrequency and switching) [1]. However, the lack of reliability feedback is at the time being one of its major drawbacks. A variety of studies were conducted in order to address those reliability questions $[2,3,4]$. DC and step stress may be interesting in order to know exactly which parameter is important on a degradation mechanism $[5,6,7]$ or with normalized tests like JEDEC in order to qualify a technology for foundries [8]. Yet, the best way to characterize the ageing of a component in its system (as a RADAR for example) is through operating conditions. With the final goal of developing a reliability guide for this technology in mind (MIL-HDBK or FIDES for example), it is mandatory to begin by determining which are the cofactors which will influence the ageing of this technology. Then, by using the Eyring law for example, it will be possible to model the operational reliability of these components depending on the junction temperature, drain bias, duty cycle of RF pulses,

In this paper, we present the results of an ageing campaign on AlGaN/GaN HEMT devices in order to analyze the effect of the drain quiescent bias on the reliability of such components in pulsed RF mode. These preliminary results allow to assess the importance of $V_{D S}$ bias for lifetime prediction in RADAR operating mode. The devices studied in this work are AlGaN/GaN HEMT power bars on $\mathrm{SiC}$ substrate with a $0.5 \mu \mathrm{m}$ gate length and $25 \mathrm{~W}$ output power. The transistor consists of $4 \times 8$ gate fingers of $250 \mu \mathrm{m}$ wide, with a source terminated field plate and air bridges. The gate to drain distance is $4 \mu \mathrm{m}$ and the pinch-off voltage is around $-2 \mathrm{~V}$.

\section{Method}

The stresses in this study are defined to be close to operational RADAR conditions. They consist in pulsed RF-HTOL ageing (Radiofrequency High Temperature Operating Life). The input power is adjusted at $35 \mathrm{dBm}$ (compression around $4 \mathrm{~dB}$, duty cycle at $10 \%$ ). One group of three devices was stressed at $V_{D S 0}=50 \mathrm{~V}$ (nominal bias, group 1) and another at $V_{D S 0}=60 \mathrm{~V}$ which is the maximum rating specified by the manufacturer (group 2). The mean junction temperature is estimated at $185^{\circ} \mathrm{C}$ for both groups using pulsed IV measurements at different temperatures and dissipated power [9]. The mean thermal resistance extracted with these electrical characterization is found at $3.54^{\circ} \mathrm{C} / \mathrm{W}$ and close to the manufacturer's value (estimated by simulation). Only the drain voltage changes between these two conditions in order to compare the parameters drifts. During the ageing, the input, output and reflected power are monitored. The average voltages and currents on the gate and the drain, as the case temperature are also recorded in order to follow a potential drift in electrical parameters. The DUTs are placed on RF amplifiers with switches used for bypassing decoupling capacitors. It is then possible to perform pulsed IV, diode and current transient measurements when the ageing is stopped. In our study, a component is considered in failure if its output power or gain is decreased by $1 \mathrm{~dB}$ or its saturated current $I_{D S S}$ and transconductance $\left(g_{m}\right)$ is lowered by $20 \%$ (values usually found in manufacturer's data). Pulsed IV measurements were performed on fresh devices at three bias points: $\left(V_{G S 0}, V_{D S 0}\right)=(0 \mathrm{~V}, 0 \mathrm{~V})$ for standard characterization $(\mathrm{P} 1),\left(V_{G S 0}, V_{D S 0}\right)=(-7 \mathrm{~V}, 0 \mathrm{~V})$ in order to have gate lag effects $(\mathrm{P} 2)$ and $\left(V_{G S 0}, V_{D S 0}\right)=(-7 \mathrm{~V}, 50 \mathrm{~V})$ for both gate lag and drain lag effects (P3). The gate lag was found to have a relatively low effect on the IV characteristics of the devices (around $4 \%$ difference between P1 and P2). On the other hand, 
drain lag has a significant importance (30\% in saturated current). This high parasitic effect is then to be followed during ageing. Measurements with A-DCTS (Athermal DC Transient Spectroscopy [10]) were performed before and after ageing in order to characterize the trapping effect evolution. These pulsed current transient measurements are conducted at different temperatures in order to extract the activation energies of defects in the device. An example of pulses sent to the gate are presented on figure 1 . The current is measured in the pulse.

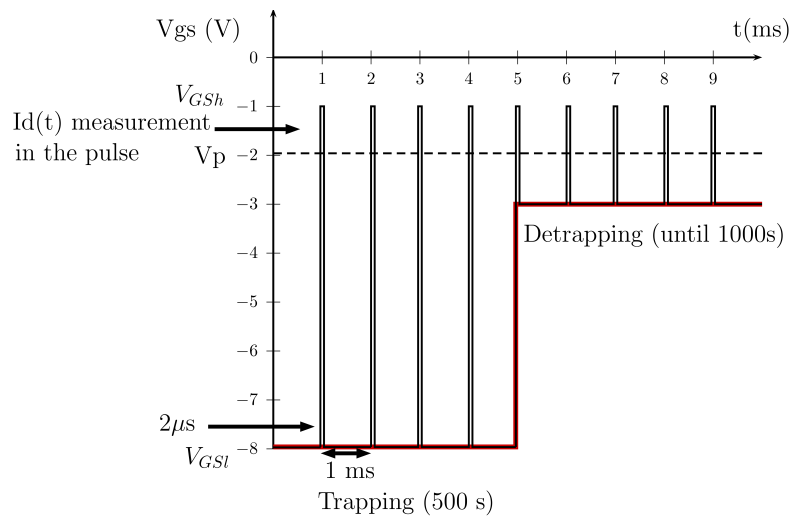

Figure 1: Examples of pulses sent to the gate of the device during A-DCTS measurement. The current measurement is done during each pulse (only the envelope is extracted)

\section{Results of the ageing tests}

During the 3500 hours of ageing, the devices in group 1 (at $\left.V_{D S 0}=50 \mathrm{~V}\right)$ show a moderate decrease in output power $(-0.5$ $\mathrm{dB}$ ). There is close to no Schottky barrier evolution (drift inferior to $5 \%$ ), indicating that the gate is relatively stable at this junction temperature. The little drop of output power seems to be due to drain lag effects as the third polarization point (P3) shows a slightly higher effect: around $8 \%$ decrease in gm. Polarizations $\mathrm{P} 1$ and $\mathrm{P} 2$ show minor variations (around 2-5\%). As expected, the second group of transistors aged at $V_{D S 0}=60 \mathrm{~V}$ manifests much higher variations than group 1 (figure 2). The output power drops by a mean of $1.7 \mathrm{~dB}$ and the Power Added Efficiency (PAE) by an average of 8.2 points and indicate the failure of these devices. As for group 1, the gate stability is validated as Schottky parameters do not vary more than $3 \%$. This stability may be due to the platinum layer included in the gate stack metalization $(\mathrm{Au} / \mathrm{Pt} / \mathrm{Ni})$ in order to reduce potential interdiffusion of metals [11].

The table 1 summarizes the evolutions of output power and PAE of the devices aged at the two different drain biases.

The peculiar PAE augmentation of device \#2 stems from the global diminution of the drain current during the RF pulse after ageing probably induced by a loss in the input impedance matching for this power amplifier, as the reflected power rises during the ageing. However, this effect is only seen for this device and comes from the drift in a RF decoupling capacitor value (not coming from the device itself). It may be noted that

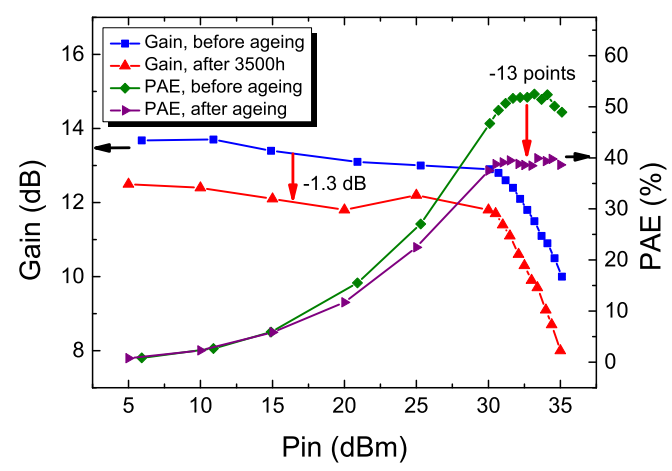

Figure 2: Gain and power added efficiency (PAE) evolution before and after $3500 \mathrm{~h}$ pulsed RF-HTOL stress, $V_{D S}=60 \mathrm{~V}$

\begin{tabular}{|c|c|c|c|}
\hline Device & $V_{D S}$ bias & $\Delta P_{\text {OUT }}(\mathrm{dB})$ & $\triangle P A E$ (points) \\
\hline$\# 1$ & $50 \mathrm{~V}$ & -0.4 & -6.1 \\
\hline$\# 2$ & $50 \mathrm{~V}$ & -0.3 & +7.4 \\
\hline$\# 3$ & $50 \mathrm{~V}$ & -0.8 & -3.3 \\
\hline$\# 4$ & $60 \mathrm{~V}$ & -2.1 & -10.3 \\
\hline$\# 5$ & $60 \mathrm{~V}$ & -1.7 & -6.1 \\
\hline$\# 6$ & $60 \mathrm{~V}$ & -1.3 & -8.2 \\
\hline
\end{tabular}

Table 1: Evolution of the output power and power added efficiency of the aged devices.

device \#2 shows relatively low RF evolution even with this drift in input impedance matching. For the other devices, the drop of efficiency stems from a significant drop in $I_{D S}$ (current collapse).

Furthermore, pulsed IV measurements at polarizations P1 and $\mathrm{P} 2$ indicate a decrease superior to $10 \%$. Then again, gate lag effect seems to have only a weak effect after ageing as the gap between $\mathrm{P} 1$ and $\mathrm{P} 2$ remains the same $(4 \%)$. However, measurements at P3 indicate a gradual diminution of $g_{m}$, until -30 $\%$ at 3500 hours (fig 3 ). The apparent recovery around $2000 \mathrm{~h}$ stems from a different measurement condition due to a hardware problem in the Pulsed IV system. The measurement had to be done again several times, with a different trapping state at its beginning. The trapping state is then not really the same as the other measurements, which explains this apparent recovery. This kind of issue was neither observed before nor after $2000 \mathrm{~h}$ of ageing and concerns only two components. Taking into account other characterizations before them, these pulsed IV measurements were performed several hours after each stop of the ageing.

It seems that drain lag effects are much more predominant than for a fresh device. Current transient measurements using A-DCTS were done on fresh and aged devices in the saturation region $\left(V_{G S h}=0 \mathrm{~V}, V_{D S}=10 \mathrm{~V}\right)$. The pulses applied to the gate allows an athermal measurement (only the signal envelope is measured). An example of transients at different temperatures is presented on figure 4 . Three exponential signatures were retrieved before and after stress, with the same activation energy 


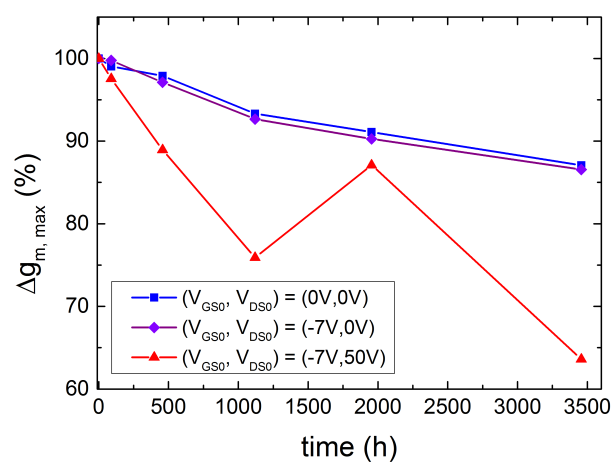

Figure 3: Normalized average decrease of the value of transconductance measured at VDS $=10 \mathrm{~V}$ for three bias points $(\mathrm{P} 1, \mathrm{P} 2$ and $\mathrm{P} 3)$. The odd point at 2000h stems from a hardware problem.

and capture cross-section (fig 5). A multi-exponential model was used for the extraction, using the Prony and LevenbergMarquardt algorithms.

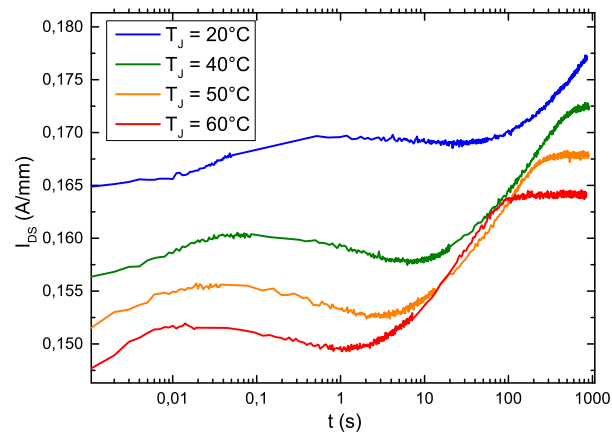

Figure 4: Example of current transients measured with A-DCTS a different temperatures for a fresh device.

Extracted activation energies are found at $\mathrm{Ea}=E_{C}-0.74 \mathrm{eV}$ (detrapping: DP1), $\mathrm{Ea}=E_{V}+0.8 \mathrm{eV}$ (trapping: TP1) and $\mathrm{Ea}=$ $E_{C^{-}} 0.65 \mathrm{eV}$ (detrapping: DP2). An activation energy close to DP1 was observed previously by Joh et. al. [12] with ON-state transient measurements. This activation energy corresponds to the dominant signature measured in our devices. According to the litterature, DP2 may be attributed to a defect linked to Fe doping in the GaN buffer [13] with the activation energy $\mathrm{Ea}=$ $0.63 \mathrm{eV}$. Finally, TP1 has been reported in the literature $[14,15$, 16] but as far as we know, its nature has not been defined to this date.

There is no difference in activation energies and capture cross-section after stress, therefore there is no formation of a new type of defect under these conditions in the measured time window of 1ms-1000s. However, the measured amplitudes associated to each time constant are much higher for two specific defects (fig 6) : $\mathrm{Ea}=E_{V}+0.8 \mathrm{eV}(\mathrm{TP} 1,+50 \%)$ and $\mathrm{Ea}=E_{C^{-}}$ $0.65 \mathrm{eV}(\mathrm{DP} 2,+30 \%)$.

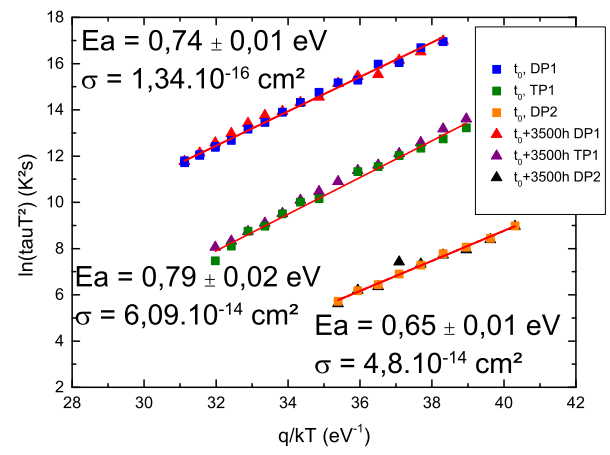

Figure 5: Arrhenius plot of the extracted traps before and after ageing using ADCTS $\left(V_{G S h}=0 \mathrm{~V}, V_{D S}=10 \mathrm{~V}\right)$. We do not detect the formation of a different defect in the $1 \mathrm{~ms}-1000$ s time scale.

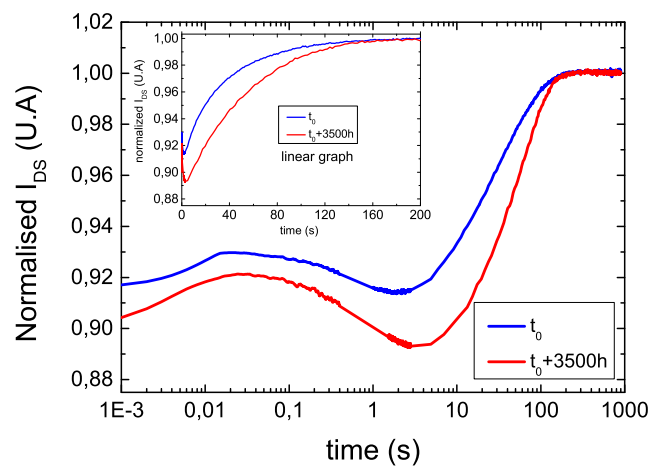

Figure 6: A-DCTS (pulsed) transients before and after ageing (3500h). With $V_{D S}=10 \mathrm{~V}$ and $V_{G S h}=0 \mathrm{~V}, \mathrm{Tc}=50^{\circ} \mathrm{C}$. Inset shows graphs in linear scale.

Additional measurements were performed in the linear region. However, trapping signatures were found to be much slower than 1000s for one of the defects, the measurement were not conclusive for this one. The signature of DP2, also visible with this polarization protocol, shows a variation of amplitude of $+35 \%$, which is in agreement with previous measurements in the saturation region.

These results are similar to [4] for long term DC stresses (gm diminution with an increased lag effect). Here, the drain voltage excursion during the RF pulse is estimated to two times the drain bias and participates in this evolution (hot carriers). The pinch-off voltage was not modified during the stresses. Only the linear region of the $I_{D S}-V_{G S}$ characteristic is changed. The degradation mechanism is attributed to traps generation induced by hot electron at the vicinity on the surface or $\mathrm{AlGaN} / \mathrm{GaN}$ interface (fig 7). This may lead to a localized diminution of the 2DEG and may explain the gradual degradation in gm.

Finally, an aged component was put aside during $800 \mathrm{~h}$, without any polarization, at ambient temperature. A final electrical characterization shows only a little recovery ( 3.5 points) in maximum transconductance. This type of degradation seems permanent as the hot electrons induced a higher concentration 


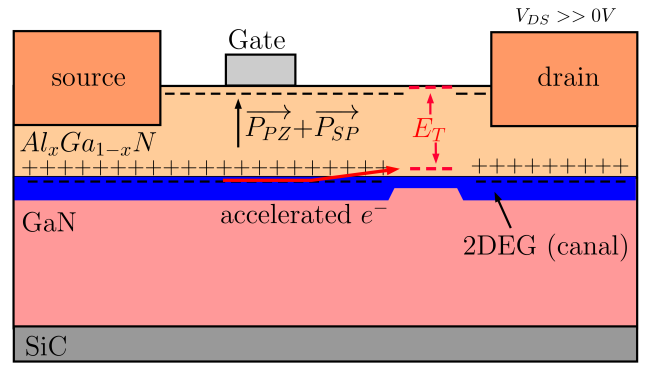

Figure 7: Assumed degradation mechanism at high drain bias during pulsed RF HTOL stress.

of deep levels in the $\mathrm{GaN}$ and $\mathrm{AlGaN}$ layers.

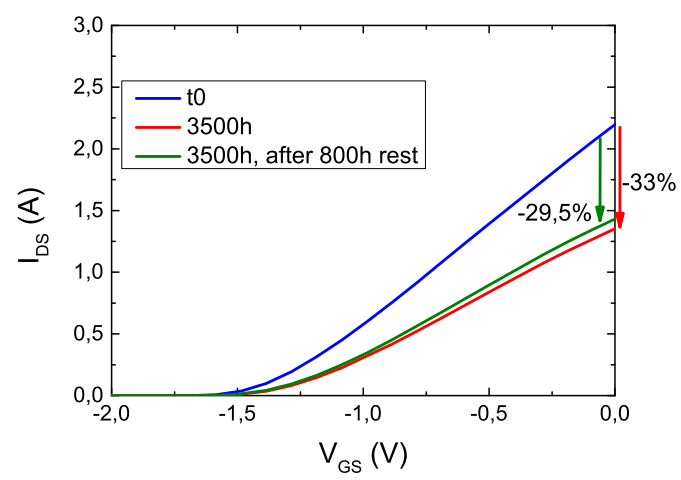

Figure 8: Transconductance measurements $\left(I_{D S}=\mathrm{f}\left(V_{G S}\right)\right.$ before, after ageing and after a $800 \mathrm{~h}$ rest. A very slight recovery indicates a rather permanent degradation.

\section{Conclusion}

Two groups of $\mathrm{AlGaN} / \mathrm{GaN}$ HEMTs on $\mathrm{SiC}$ substrate were stressed in pulsed RF-HTOL conditions, with the same estimated junction temperature $\left(185^{\circ} \mathrm{C}\right)$. During these stresses, the increase in drain quiescent voltage leads to higher trap generation, resulting in a diminution of gm and $I_{D S S}$. The high drain voltage excursion during the RF pulse intensifies the hot carriers effect leading to trap generation for defects at $\mathrm{Ea}=E_{V}+0.8$ $\mathrm{eV}$ and $\mathrm{Ea}=E_{C^{-}}-0.65 \mathrm{eV}$ in these conditions. These defects are assumed to be in $\mathrm{GaN}$ buffer especially for $\mathrm{Ea}=E_{C^{-}}-0.65$ $\mathrm{eV}$. The degradation seems permanent as after a $800 \mathrm{~h}$ rest period, only a slight recovery in transconductance is recovered. No difference in pinch-off voltage was measured. Diode measurements indicates that the gate is stable under these conditions (drift $<5 \%$ ), which may be due in part to the platinum layer under the gate stack metalization $(\mathrm{Au} / \mathrm{Pt} / \mathrm{Ni})$. These results confirm the importance of drain voltage as an acceleration factor for ageing in pulsed RF condition. Further work on the impact of RF pulses duty cycle is planned in the future.

\section{ACKNOWLEDGEMENT}

The authors would like to thank the french Department of Defense (DGA) for its financial support to this work.

\section{REFERENCES}

\section{References}

[1] Y. F. Wu, M. Moore, A. Saxler, T. Wisleder, and P. Parikh. 40-W/mm Double Field-plated GaN HEMTs. In Device Research Conference, 2006 64th, pages 151-152. IEEE, June 2006.

[2] J. B. Fonder, L. Chevalier, C. Genevois, O. Latry, C. Duperrier, F. Temcamani, and H. Maanane. Physical analysis of Schottky contact on power AlGaN/GaN HEMT after pulsed-RF life test. Microelectronics Reliability, 52(9-10):2205-2209, September 2012.

[3] J. A. del Alamo and J. Joh. GaN HEMT reliability. Microelectronics Reliability, 49(9-11):1200-1206, September 2009.

[4] G. Meneghesso, G. Verzellesi, F. Danesin, F. Rampazzo, F. Zanon, A. Tazzoli, M. Meneghini, and E. Zanoni. Reliability of GaN HighElectron-Mobility Transistors: State of the Art and Perspectives. Device and Materials Reliability, IEEE Transactions on, 8(2):332-343, June 2008.

[5] D. Marcon, J. Viaene, P. Favia, H. Bender, X. Kang, S. Lenci, S. Stoffels, and S. Decoutere. Reliability of AlGaN/GaN HEMTs: Permanent leakage current increase and output current drop. Microelectronics Reliability, 52(9-10):2188-2193, September 2012.

[6] Valerio Di Lecce, Michele Esposto, Matteo Bonaiuti, Gaudenzio Meneghesso, Enrico Zanoni, Fausto Fantini, and Alessandro Chini. Experimental and simulated de degradation of GaN HEMTs by means of gate-drain and gate-source reverse bias stress. Microelectronics Reliability, 50(9-11):1523-1527, September 2010.

[7] Y. C. Chou, D. Leung, I. Smorchkova, M. Wojtowicz, R. Grundbacher, L. Callejo, Q. Kan, R. Lai, P. H. Liu, D. Eng, and A. Oki. Degradation of $\mathrm{AlGaN} / \mathrm{GaN}$ HEMTs under elevated temperature lifetesting. Microelectronics Reliability, 44(7):1033-1038, July 2004.

[8] B. Lambert, J. Thorpe, R. Behtash, B. Schauwecker, F. Bourgeois, H. Jung, J. Bataille, P. Mezenge, C. Gourdon, C. Ollivier, D. Floriot, and $\mathrm{H}$. Blanck. Reliability data's of 0.5 ? $\mathrm{m} \mathrm{AlGaN} / \mathrm{GaN}$ on $\mathrm{SiC}$ technology qualification. Microelectronics Reliability, 52(9-10):2200-2204, September 2012.

[9] Jungwoo Joh, Jesús del Alamo, Uttiya Chowdhury, Tso-Min Chou, HuaQuen Tserng, and Jose L. Jimenez. Measurement of Channel Temperature in GaN High-Electron Mobility Transistors. Electron Devices, IEEE Transactions on, 56(12):2895-2901, December 2009.

[10] A. Divay, M. Masmoudi, O. Latry, C. Duperrier, and F. Temcamani. An athermal measurement technique for long time constants traps characterization in GaN HEMT transistors. Microelectronics Reliability, 55(910):1703-1707, August 2015.

[11] Helmut Jung, Reza Behtash, James R. Thorpe, Klaus Riepe, Franck Bourgeois, Hervé Blanck, Andrey Chuvilin, and Ute Kaiser. Reliability behavior of GaN HEMTs related to Au diffusion at the Schottky interface. physica status solidi (c), 6(S2):S976-S979, June 2009.

[12] Jungwoo Joh and Jesus Del Alamo. A Current-Transient Methodology for Trap Analysis for GaN High Electron Mobility Transistors. Electron Devices, IEEE Transactions on, 58(1):132-140, January 2011.

[13] Gaudenzio Meneghesso, Matteo Meneghini, Davide Bisi, Isabella Rossetto, Andrea Cester, Umesh K. Mishra, and Enrico Zanoni. Trapping phenomena in AlGaN/GaN HEMTs: a study based on pulsed and transient measurements. Semiconductor Science and Technology, 28(7):074021+, July 2013.

[14] A. Y. Polyakov, N. B. Smirnov, A. S. Usikov, A. V. Govorkov, and B. V. Pushniy. Studies of the origin of the yellow luminescence band, the nature of nonradiative recombination and the origin of persistent photoconductivity in n-GaN films. Solid-State Electronics, 42(11):1959-1967, November 1998.

[15] G. A. Umana-Membreno, G. Parish, N. Fichtenbaum, S. Keller, U. K. Mishra, and B. D. Nener. Electrically Active Defects in GaN Layers Grown With and Without Fe-doped Buffers by Metal-organic Chemical Vapor Deposition. Journal of Electronic Materials, 37(5):569-572, 2008.

[16] W. Götz, J. Walker, L. T. Romano, N. M. Johnson, and R. J. Molnar. Thickness Dependence of Electronic Properties of GaN Epi-layers. In 
Symposium N-III-V Nitrides, volume 449 of MRS Online Proceedings Library, 1996. 